\title{
The Research of the Leisure Agriculture Park Based on Horticultural Therapy*
}

\author{
Yufan Ding \\ College of Landscape Architecture \\ Sichuan Agricultural University \\ Chengdu, China
}

\author{
Hong Zhang \\ College of Landscape Architecture \\ Sichuan Agricultural University \\ Chengdu, China \\ Lei Lei \\ College of Landscape Architecture \\ Sichuan Agricultural University \\ Chengdu, China \\ Xiaoyu Ming \\ College of Landscape Architecture \\ Sichuan Agricultural University \\ Chengdu, China
}

\author{
Yan Feng \\ College of Landscape Architecture \\ Sichuan Agricultural University \\ Chengdu, China \\ Panpan Cao \\ College of Landscape Architecture \\ Sichuan Agricultural University \\ Chengdu, China \\ Xiaofang $\mathrm{Yu}^{* *}$ \\ College of Landscape Architecture \\ Sichuan Agricultural University \\ Chengdu, China \\ **Corresponding Author
}

\begin{abstract}
As a medical method integrating treatment, rehabilitation and vocational training, horticultural therapy is same as other treatments like music therapy and art therapy all belonging to the category of occupational therapy. Horticultural therapy is for people who need physical and mental improvements, which is an effective way to adjust with activity of plant cultivation and horticultural operation from its social, education and mental and physical aspects. Through gardening, people can build up their bodies, eliminate frustrations, improve their lives and mental health, and perceive the fruits of their labor and the joy they bring. With the development of society and the accumulation of practice, horticultural therapy has been paid more and more attention. In developed countries, many mental hospitals, rehabilitation institutions, intellectual disability education centers and other units are equipped with such treatment services. Even for the general population's spiritual needs, there are many botanical gardens, gardens and so on.
\end{abstract}

Keywords-horticultural therapy; leisure agriculture park; healing garden; agritainment

\section{INTRODUCTION}

Horticultural therapy belonging to horticultural activities

*Thanks for Guangdong provincial science and technology project (2017B090907001). in leisure agricultural gardens can combine medicine with leisure agriculture, which is a new development trend of leisure agriculture. In the construction of leisure agriculture garden, horticultural therapy can relieve people's pressure and enjoy life through various characteristics and habits of plants, and also can treat people's body and mind. The leisure agriculture park studied in this paper lies between the general leisure agriculture park and the special gardening therapy garden, which is the organic combination of the two. Generally speaking, there is no special area for gardening activities and gardening appreciation in the leisure agricultural park, where the visitors are not very participatory and the population is not very targeted. However, horticultural therapy is mainly used for the treatment of patients, and the target group is too small to meet the needs of ordinary people. Based on the investigation and study of the existing leisure agricultural park cases, this paper puts forward the idea of innovative design combining with the design method of horticultural therapy, which is applying the theory and strategy of horticultural therapy in the planning and design of leisure agriculture park to achieve the purpose of promoting people's healthy. As well as in the allocation of plants to make full use of the plant in horticultural therapy, so that the effects of plants on human rehabilitation can be brought into play. 


\section{OVERVIEW OF HORTICULTURAL THERAPY}

\section{A. The Definition of Horticultural Therapy}

Horticultural Therapy is a new medical method, which is the same as music therapy and art therapy. On the basis of summarizing various definitions, $\mathrm{Li}$ Shuhua defined horticultural therapy as an effective method to maintain and restore people's physical and mental functions and improve the quality of life through plants, the growing environment of plants and various activities related to plants. In simple terms, horticultural therapy is to use horticulture to treat people who need to improve their physical and mental health. The main way is to use plant cultivation and horticulture activities to adjust from their social, education, psychological and physical aspects. People in gardening activities can not only improve their physical fitness, eliminate frustrations, and promote mental health, but also feel the fruits of their labor, thus feeling happy. The interaction between humans and plants not only promotes healing, but also relaxes the body and gives pleasure.

\section{B. The Types of Horticultural Therapy}

1) Environmental treatment: Environmental treatment mainly refers to that various sensory factors that can stimulate human body in the environment can act on human body to make people comfortable and thus achieve the therapeutic effect. It mainly includes the following sensory stimulation factors.

a) Tactile stimulation: Different textures of plants can give different senses, and different parts of plants can stimulate different senses.

b) Auditory stimulation: Different forms and different types of plant leaves collide in the wind and rain. This beautiful sound from plants can calm and eliminate irritability.

c) Visual stimulation: Leaves of different shapes and colors bring different visual effects.

d) Smell stimulation: Different fragrance of plants can stimulate different smell, people stop in the garden full of fragrance, feeling extremely relaxed and comfortable.

e) Taste stimulation: Create fruits, vegetables and herbs by hand and share them with others, giving the grower a sense of accomplishment.

2) Playing treatment: Through the amusement facilities for tourists can let them play in the beautiful environment, resolve problems in the process, eliminate boredom, enhance curiosity and confidence, this way is called play therapy.

3) Horticultural treatment: The Lack of physical exercise leads to physical decline, which leads to mental decadence, low physiological function and negative mental state. Horticulture treatment provides people with the opportunity to practice and improve their confidence and health through a series of activities such as land clearing, fertilizing, sowing, management and harvesting. This is called horticulture therapy.

4) Memory treatment: It's based on the design model of the American Landscape Architect Robert Hoover.
According to the theory, the three stages (early, middle and late) of Alzheimer's disease correspond to the three stages (late, middle and early) of normal growth, and the courtyard design should meet the needs of patients at different stages. For example, early stage patients, equivalent to the later stage of normal growth, like independence, adventure and freedom. The design can be artificially provide activity area, such as stadiums, amusement parks, planting pool for exercise, or set covered by finely pebbles, relatively steep stone road as a "challenge road", satisfy their lust for adventure, arouse their memories of the past.

\section{The Efficacy of Horticultural Therapy}

Professor Li Shuhua, a famous expert in horticultural therapy research in China, has summarized its efficacies in social, physical, spiritual and technical aspects. In the social aspect, it has the function of improving social ability and public morality. In the physical aspect, there is the effect of stimulating the senses and strengthening the motor function. In terms of spirit, it has the effect of increasing vitality, elevating atmosphere, inhibiting impulsiveness, enhancing sense of responsibility, building self-confidence, cultivating creativity, building endurance and attention, eliminating restless psychology and impatience, acting with planning and full of confidence for the future. In terms of skills, there is the effect of vocational training and social contribution. In recent years, with the popularization of horticulture therapy, it has been applied in the recreational agricultural park. The author believes that horticultural therapy can exert effects on people's physical and mental health, enhance social awareness, strengthen physical and mental health and cultivate feelings.

\section{The Elements of Horticultural Therapy}

Horticultural therapy is an activity centered on the cultivation and appreciation of plants. People establish a close relationship with plants during the process of participation, and feel the growth process of plants and the benefits brought by plants to the body and mind. Therefore, the three elements that constitute horticultural therapy are: plants, various activities related to plants and the habitat of plants.

1) Plant factor: Plants are alive. People feel the passage of time and the change of seasons in the process of cultivating plants, and they have improved their confidence after seeing the fruits of their labor. In addition, many volatile substances from plants have health functions for people and regulate human function.

2) Activity factor: The difference between horticultural therapy and other therapies is that horticultural therapy is a kind of activity centered on growing plants, which can bring people pleasure from the whole process of growing plants to the final harvest and use.

3) Environment factor: People can experience the changes of season, climate and natural environment directly when they participate in gardening therapy activities. From the moment they began to cultivate the plants, they have gradually established a connection with the nature, and the 
growth of plants also restored people's life rhythm. People communicate and cooperate with each other in a relaxed state in a variety of plant environments, so that the mood is calmed, the worries and pains of reality are gradually forgotten, and communication and self-cognition are improved at the same time.

\section{THE OVERVIEW OF LEISURE AGRICULTURE PARK}

\section{A. The Definition of Leisure Agriculture}

Leisure Farming originated from the Leisure industry, which is an industry providing Leisure services. There are many related nouns about leisure agriculture, mainly including: rural tourism, leisure agriculture tourism, sightseeing agriculture, holiday farm, leisure farm, rural tourism, rural tourism, agricultural tourism, etc. The author thinks that the leisure farm is a new type of green industry model, it makes full use of rural human resources and natural resources, through a series of planning and construction to develop the function of leisure agriculture, tourism, to provide tourists sightseeing tourism, leisure activities, let people fully experience the rural and agricultural and relax body and mind and relieve pressure, so as to improve the quality of life.

\section{B. The Main Types of Leisure Agriculture Parks}

The author takes comprehensive consideration of leisure content, activity projects, sightseeing and research contents of this paper, and divides the leisure agriculture park into several types, including agricultural trade type, agricultural garden type, agritainment type, characteristic agricultural industry type, and leisure farm type.

1) Agricultural trade type: A leisure agricultural park, which allows visitors to purchase agricultural and sideline products and services while sightseeing, needs to rely on the distribution centers of large and medium-sized agricultural and sideline products.

2) Agricultural garden type: Through the promotion, demonstration and popularization of advanced modern science and technology and agronomy technology, this leisure agriculture park can show people the elegant demeanor of modern agriculture while providing high-tech agriculture sightseeing, high-quality agricultural products procurement, agricultural knowledge education, tasting and picking and other recreational products.

3) Agritainment type: This type of leisure agriculture park is built on the basis of the original rural villages, residential buildings and rural culture. Tourists can not only enjoy the original rural dishes in the ordinary rural courtyards, but also experience the local customs and practices of honest rural areas.

4) Characteristic agricultural industry type: This leisure agricultural park makes full use of modern cultivation techniques and management results, and relies on the advantageous agricultural industrial belt and experience activities such as picking and pruning to deeply explore the tourism value of agricultural landscape. Tourists can visit, pick and shopping here.

5) Leisure farm type: On the basis of the original modern agricultural park, relying on the local beautiful scenery, fresh and pleasant climate, and green ecological environment, leisure and entertainment facilities are established to provide tourists with leisure vacation, entertainment and accommodation services, so that people can enjoy the quiet and comfortable countryside and truly return to the nature.

\section{THE RELATIONSHIP BETWEEN HORTICULTURAL THERAPY AND LEISURE AGRICULTURE PARK}

Horticultural therapy belongs to horticulture activities in leisure agriculture garden. It can combine medicine with leisure agriculture and is a new development trend of leisure agriculture. In the construction of leisure agriculture park, horticultural therapy can relieve people's pressure and enjoy life through various characteristics and habits of plants, and also can treat people's body and mind at the same time. The leisure agriculture park studied in this paper lies between the general leisure agriculture park and the special horticultural therapy garden, which is the organic combination of the two. In general, there is no special area for gardening activities and gardening appreciation in the leisure agricultural garden, so the participation of visitors and the targeted population are not strong. Horticultural therapy is mainly used for the treatment of patients, and the target group is too small to meet the needs of ordinary people. This paper mainly studies based on gardening therapy as a guide the design of leisure agriculture park, combination of plant landscape with a variety of plant experience activities as the focus of the park construction, production, leisure, entertainment, and the residents as the theme, for people to watch, to participate in the activities of unique experience and popular science education, rehabilitation, the perfect combination of sports and leisure tourism, so as to find the better means of attracting tourists.

\section{CASE StUdy OF HORTICULTURAL THERAPY ABROAD}

The habitat of the mind - a reference to the design concept and techniques of Joe Delaney:

\section{A. The Design Philosophy of Joe Delaney}

1) The philosophical thinking of gardens: Influenced by philosophy and human culture, Delaney often ponders the origin of garden design. Why do we design gardens? What do we want the garden to tell us? Delaney tries to answer her questions about "source" through the garden design.

2) The narrative concept of garden: Delaney believed that one could find a temporary peace of mind in the garden, free from outside disturbance, and could use it as a medium for the transmission of the human spirit. Therefore, she USES the experience of garden users as a source of creation.

3) The sense of garden experience: Delaney attaches great importance to the user's visual, tactile, auditory and olfactory sensory experiences in the garden design. These 
sensory experiences can give rise to colorful time and space and make the landscape full of changes.

\section{B. Joe Delaney Design Technique}

1) Visual construction: Through the design of hard landscape and the application of colors, more layers and imagination of vision are provided; see "Fig. 1" and "Fig. 2".

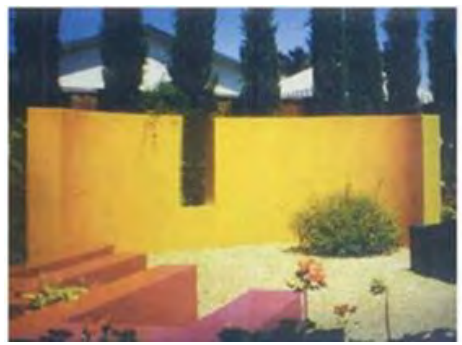

Fig. 1. Gustav garden.

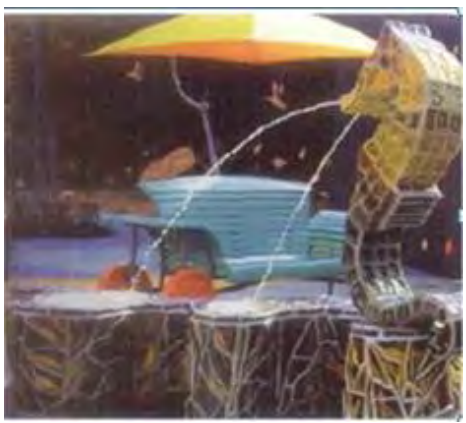

Fig. 2. Seahorse fountain.

2) Tactile construction: Through the application of textured materials, such as stainless steel, concrete, ground glass and gravel, people can feel industrial civilization; see "Fig. 3" and "Fig. 4".

3) Creation of hearing: Mainly through the sound of water flow and the swaying sound of plants, such as the deep and persistent sound after the water in the water container of Xie Garden "Fig. 5", and the rustle of the bamboo in Tianqi Garden "Fig. 6" when the wind blows, all bring the quiet and peaceful atmosphere to visitors.

4) Creation of sense of smell: Through fragrant plants such as gardenia, lavender, thyme, rose, etc.

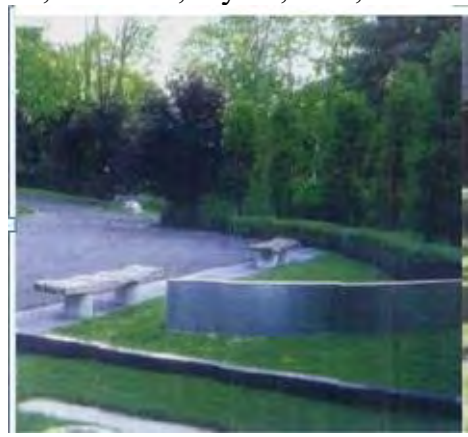

Fig. 3. Wall stainless.

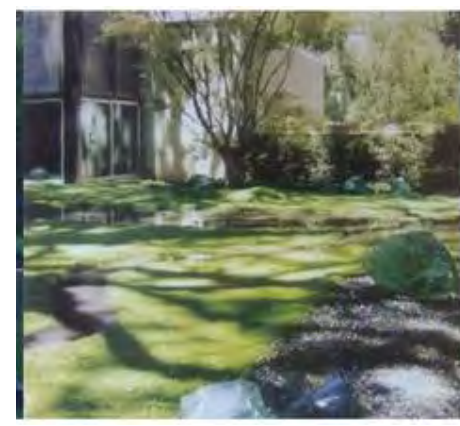

Fig. 4. Ground glass and balls steel with concrete.

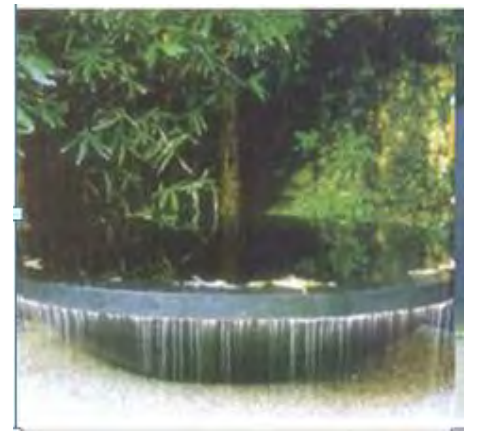

Fig. 5. Water container in Xie Garden.

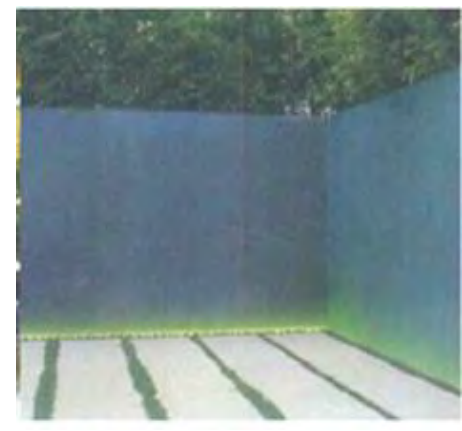

Fig. 6. Su bamboo in Tianqi Garden.

\section{CASE STUDY OF DOMESTIC LEISURE AGRICULTURAL PARK}

\section{A. Analysis of Current Situation of Leisure Agricultural} Park - Taking Sichuan Province as an Example

As a large agricultural province with a large population, the rural music industry in Sichuan province has developed to a considerable scale. In recent years, the leisure agriculture and rural tourism, supported by the agritainment, have been developing vigorously and gradually become the emerging industry that promotes the increase of farmers' employment and the development of modern agriculture. From the emergence of the first generation of the agritainment in the $1980 \mathrm{~s}$ to the second generation of "nongjiale" in the 1990s and now to the third generation of the agritainment, it has experienced nearly 20 years of development and changes.

1) Rural landscape model - agricultural village in Pidu district: Agricultural village is located in Pidu district 
in Chengdu city, Sichuan province, is the national agricultural tourism demonstration site, is the birthplace of "agrtainmentl", known as "flowers village, fenceless park". Here, you can enjoy the rural gardens and bonsai art, experience the development process of rural music, enjoy the rural catering, buy rural featured goods, and experience rural folk customs. In the Chinese bonsai garden in agricultural village, there are five areas of ginkgo biloba, golden marble, wintersweet, luohansong pile and exquisite bonsai in the garden of more than $20 \mathrm{mu}$. Each area of the high and low scattered, the wind and posture each beautiful, mutual cover light. The layout of the garden is rigorous and there is no lack of activity, the circuitous and winding roads lead to seclusion. The restaurant covered with wheat straw, the tea gallery with pure bamboo structure, and the numerous outstanding root carving with various gestures constitute a vivid folk painting of western Sichuan "Fig. 7" and "Fig. 8".

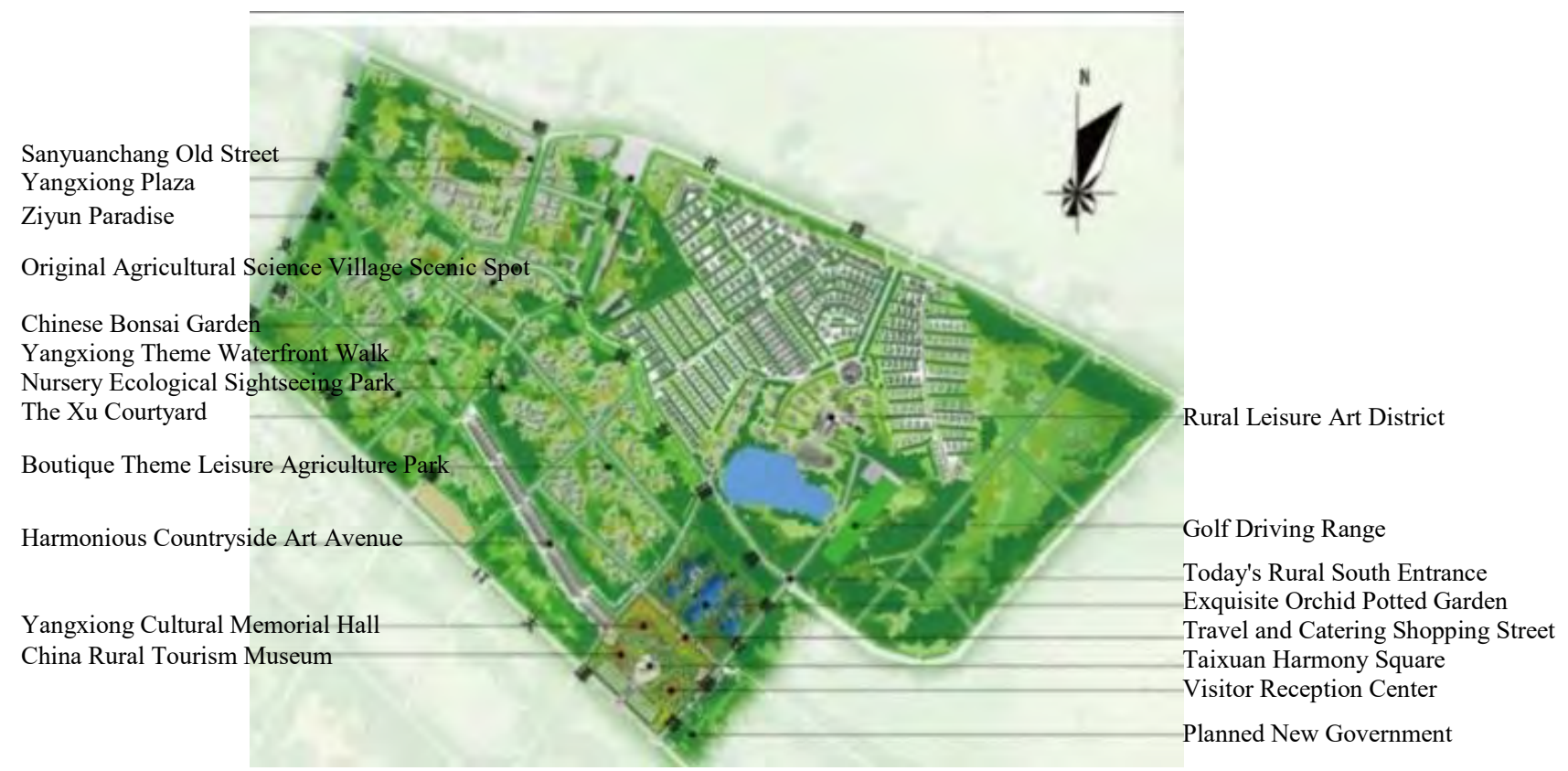

Fig. 7. Agricultural village planning general plan.

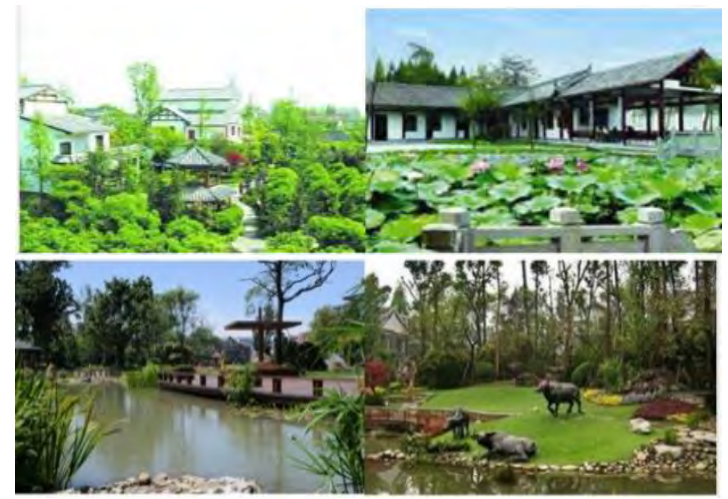

Fig. 8. Agricultural village photos.

2) The pattern of watching flowers and fruits represented by agritainments in Longquanyi district, Chengdu: To view peach (pear) flowers in the spring, summer fruit sightseeing tour, so that people first feast their eyes, and fill their mouth. For example, while taking a vacation, tourists can also go to the orchard to pick fruit and taste fruit, which has a unique rural flavor "Fig. 9" and "Fig. $10 "$.

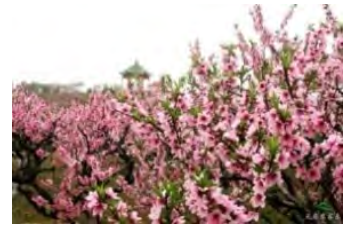

Fig. 9. Peach blossom in Longquanyi district.

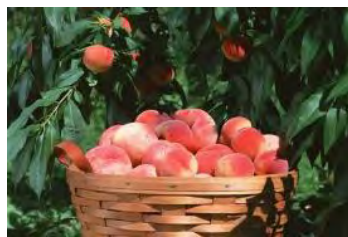

Fig. 10. Peach in Longquanyi district.

3) Self-entertainment and self-music mode - some agritainments in Huayang town, Shuangliu district is represented: For example, tourists can enjoy the fun of fishing in a picturesque garden instead of being busy and annoyed. In addition, some rural families have introduced the experience service of personally experiencing the rural life. People can personally feel the mild climate, fresh air, fertile soil, simple folkway and harmonious life, so that people can get away from the hustle and bustle of the city and fully 
integrate into the rural life. Visitors can not only get a lot of knowledge about rural life, but also go to the fields to cultivate, select vegetables and cook by themselves. Here, you can experience the unique farmhouse joy, taste authentic country food and write your own idyllic poem.

4) Featured theme mode - represented by "five golden flowers" in Jinjiang district, Chengdu: "Five golden flowers" refers to the five villages under the office of Sansheng street in Jinjiang district, Chengdu, including the rural residence of Huaxiang — red sand village "Fig.11", happy merlin - Xingfu village "Fig.12", Jiangjia vegetable field - Jiangjiayan village "Fig.13", Donglijuyuan - Fuma village "Fig.14", and Moonlight of the lotus pond - Wanfu village "Fig.15". Jinjiang district started to build the agritainments here at the beginning of 2003. After three years of efforts, it has become an open park full of rural scenery, a sample of national rural tourism scenic spots, and a model combining urban-rural planning with tourism development. Now, "five golden flowers" conducts dislocation development and race to the open. "The rural residence of Huaxiang" has become a national AAAA class scenic spot dominated by the development of small POTS, fresh cut flowers and tourism industry. "Jiangjia vegetable field" is a place for tourists to recognize and cultivate land and vegetables, enjoy the joy of harvest, experiencing the pleasant and leisure, sweat and toil of rural life. "Donglijuyuan" highlights multiple species of chrysanthemum and large scale of chrysanthemum garden, forming the integration of "environment, humanity, chrysanthemum rhyme and flower sea". "Moonlight of the lotus pond" has become an art village of art creation and development of music.

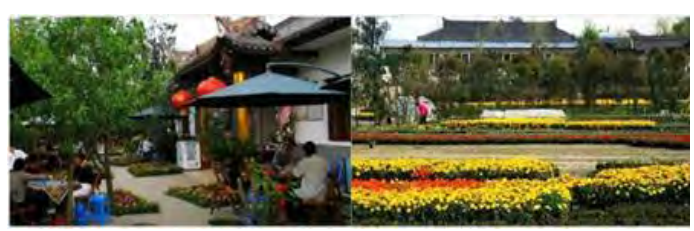

Fig. 11. Red sand village.

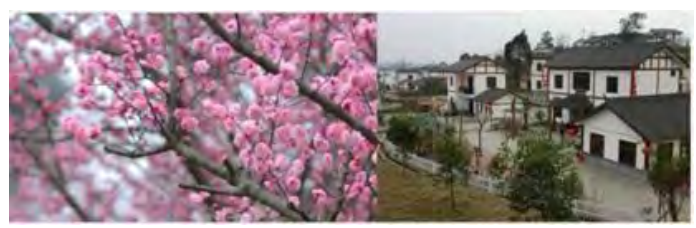

Fig. 12. Xingfu village.

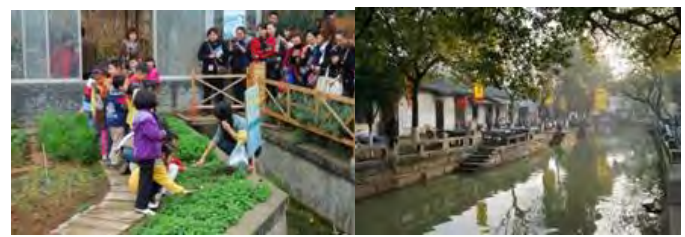

Fig. 13. Jiangjiayan village.

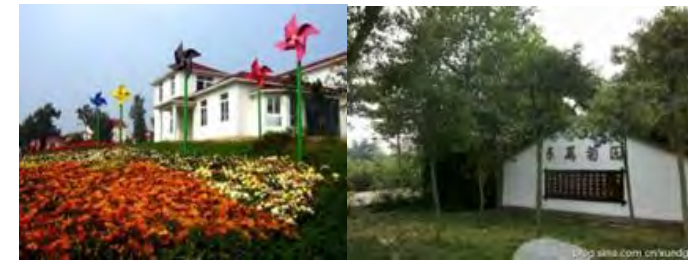

Fig. 14. Fuma village.

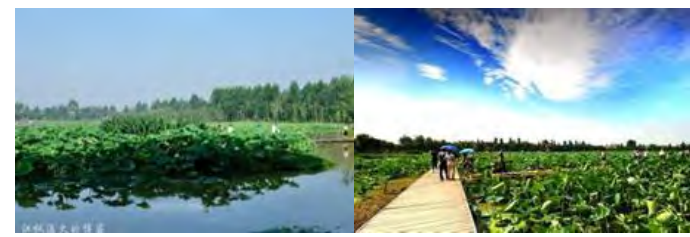

Fig. 15. Wanfu village.

\section{B. Case Analysis Inspiration}

This paper discusses the planning and design of the leisure agricultural garden mainly based on horticulture therapy, which involves the majority of urban residents, meets the needs of all kinds of people, and can experience the efficacy of horticulture therapy whether healthy or not. Based on the analysis of the current situation of the leisure agricultural parks in China, this paper summarizes the following points that deserve more attention in the future.

- Meeting functional requirements while paying attention to the humanized design: "Humanized design" has become a popular trend of contemporary landscape design, but we often look at the laying of guide tiles, barrier-free ramp, and braille signs. When we deliberately add these facilities in the landscape design, although they facilitate the disabled, but rigidly and abruptly divide the disabled clearly, which itself loses the basic meaning of humanized design.

- Giving priority to plant landscape design, a few hard landscape design and hard landscape design and paying attention to details and humanization

- Paying attention to the sense of scale of the venue and facilities to meet the experience needs of all kinds of people

- Using the space partition and boundary enclosure to create open and private space to meet the needs of different tourists

- Making full use of visual, auditory, olfactory, tactile and other sensory stimulation, selecting the natural factors that bring stimulation with pertinence, creating rich landscape for tourists, and achieving the purpose of appreciation, leisure and health.

\section{DESIGN PRACTICE OF LEISURE AGRICULtURE GARDEN BASED ON HORTICULTURAL THERAPY}

From previous theoretical studies, we have learned that one of the most important factors of horticultural therapy is plants, which can achieve therapeutic or rehabilitation effects on human body through various horticultural activities by plants themselves or around them. Incense therapy, forest 
bathing, color therapy, landscape therapy, air anion therapy, all of these therapies belong to the field of horticulture therapy, which are the implementation of auxiliary horticulture therapy to achieve better therapeutic effect. Through literature and case study, the implementation plan of horticulture therapy is summarized, in order to provide reference for future design practice of horticulture therapy in leisure agriculture.

\section{A. Specific Implementation Plan of Horticultural Therapy in Leisure Agriculture Parks}

1) Hiring a horticulture therapist and training volunteers: With the help of volunteers, horticulture therapists sorted out the available resource environment according to the requirements of horticulture therapy, conducted a comprehensive survey on the development status of local horticulture therapy and the understanding of people, and then developed a reasonable project plan according to the professional development requirements.

2) Making a list of the participants: Horticulture therapists designed and produced questionnaires that could reflect the participants' own situation, and then asked the participants to fill them out according to their own characteristics and mental status, as the basis for the evaluation of the results, and the ultimate goal to be achieved. According to the above situation, the amount of labor and content of gardening activities will be suitable for the physical condition of participants.

3) Collaborating with volunteers: As an assistant to the horticulture therapist, the volunteer must establish a good cooperative relationship in order to better guide the participants in various gardening activities. At the same time, during the process of assisting the horticulture therapist, the volunteers must record the participants' impressions and points of attention in detail, which can be used as an important basis for the post-event evaluation.

4) Making the process of gardening fun and easy to understand: During the implementation of the program, participants' motivation and emotions fluctuate with the activities. Therefore, the program design must be interesting to arouse the participants' enthusiasm and ensure the sustainability of gardening activities.

5) Recording activity status and results: Horticulture therapists record the process and results of activities in the form of words or photographs. At the same time, participants need to record their feelings and self-evaluation during the process, which also improves their self-recognition and perception of things.

6) Checking and evaluating the activity status: During the implementation of horticulture therapy, horticulture therapists evaluated the activity status and progress of participants through self-observation, recording and recording of participants themselves, and then preliminarily speculated and evaluated the effect of the procedure.

\section{B. Vision of the Future}

The design of leisure agriculture garden based on horticultural therapy is a relatively new topic and needs to be studied in a complex way.

1) Learning and drawing on horticultural therapy technology: Through gardening, people can build up their bodies, eliminate frustrations, improve their lives and mental health, and perceive the fruits of their labor and the joy they bring. The application of horticulture to human health has been documented in many countries, although the use of it in recreational agricultural gardens is rare. However, with the development of social civilization and the accumulation of practice, more and more attention has been paid to the innovative forms of the combination of gardening therapy and leisure agriculture.

2) Establishing a sound discipline system, practice methods, rules and regulations: Only when gardens, cities and people depend on each other and integrate into each other, can we fully meet the needs of human society for survival and development, build the ecological community of health care, and provide people with a living and working space with sound ecology, good air and beautiful environment. To sum up, in the design of the leisure agricultural garden for horticultural recuperation, the emphasis on the curative effect of horticultural therapy and the basic principles of its application are the fundamental needs of fully reflecting humanization. Combined with the advantages of the leisure agricultural garden, it creates unique experience for people, which is also the fundamental trend of garden construction in the 21 century.

3) Strengthening the discipline construction of horticultural therapy: Horticultural therapy itself is a new discipline, facing many difficulties, but horticulture therapy for people's physical and mental treatment is positive, the application of horticulture therapy in the recreational agricultural garden to the treatment of physical and mental illness and spiritual entertainment has a broad prospect. Due to the limited conditions, only foreign cases were selected for the investigation and analysis of horticultural therapy in this paper. However, there were few actual cases in China. It is hoped that in the follow-up study, the actual cases can be studied to obtain the more comprehensive application status of gardening therapy in China, so as to guide the design of leisure agriculture garden with gardening therapy in various regions.

4) Examining the reality and rationality of horticultural therapy in practice: At present, as far as Sichuan province is concerned, there are a large number of leisure agricultural parks, and the leisure agriculture industry is flourishing. The design project of leisure agriculture garden based on horticultural therapy and the approach to implement horticultural therapy proposed in this paper were summarized based on relevant literature research and the reference of foreign excellent cases. Whether it can reflect rationality in reality still needs to be tested. 


\section{CONCLUSION}

Nowadays, ecological agriculture is developing rapidly. With the increasing social competition, people pay more and more attention to "gardening therapy" as a way to relieve pressure and promote health. Therefore, it is of great significance to integrate horticultural therapy into the design of leisure agricultural gardens.

Through collecting and reading, this study sorted out the leisure agriculture park and horticultural therapy. Then select two typical leisure agriculture parks as an example, and through field visits, surveys, function layout, characteristics of choosing area, plant landscaping, characteristic tree species, the degree of greening, facilities and so on has carried on the investigation and analysis, sums up their success and failure, with reference of foreign outstanding case, based on the planning and design of the leisure agriculture park of horticultural therapy, summarized the theory, And to find suitable horticultural therapy into the leisure agricultural park design ideas. Under the background of the new era, the horticultural therapy and leisure agriculture garden itself can get better development. Meanwhile, they will combine more closely and promote related research development.

\section{REFERENCES}

[1] Li Shuhua. Introduction to Horticulture Therpy [M]. China Forestry Press, 2011.

[2] Li Shuhua. Early Establishment of a Discipline System of Horticultural Therapy with Chinese Characteristics [J]. Chinese Garden, 2000(3): 17-19.

[3] Weng Mei. Auditory Landscape Design [J]. Chinese Garden, 2007, 12:46-50.

[4] Li Kaidong. Weihong Wang. Environmental Perception and Color Environment Design of Medical Architecture [J]. Journal of West College of South China Construction University, 1999,7 (2) : 76-81.

[5] Song Zhumao. Design Psychology [M]. Guangzhou: Guangdong Higher Education Press, 2007:27-33.

[6] Jan Galle. Translation by Renke He. Communication and Space [M]. Beijing: China Construction Industry Press, 2002:68-73.

[7] Qi Daiwei. Achieving Physical and Mental Balance -- Preliminary Exploration of Rehabilitation and Recuperation Space Landscape Design [D]. Beijing: Beijing Forestry University, 2007.

[8] (Taiwan) Guo Yuren. Theory and Operation Manual of Horticulture and Landscape Therapy [M]. Taiwan: Landscape Studies, China Culture University, 2002:20-52

[9] Lindquist. Therapy Through Play [M ]. London: Arlington Books, 1977.

[10] Hoover R. Healing gardens and alzheimer's[J ]. The American Journal of A lzheimer's Care and Related Disorders and Research, 1995, 13 (4) : 129

[11] Qi Yunzhi. Tianshou Xie and Yongjun Du. Construction of Ecological Community of Health Care Type in Urban Gardens [J]. Chinese Landscape Architecture, 2003,19 (10):31-33.

[12] Lin Yulian. Zhengfan Hu. Environmental Psychology (Second Edition)[M]. Beijing: China Architecture Industry Press, 2006.

[13] Qi Daiwei. Achieving Physical and Mental Balance -- Preliminary Exploration of Rehabilitation and Recuperation Space Landscape Design [D]. Beijing: Beijing Forestry University,2007.

[14] Bao Zhibo. A Brief Analysis of the Application of Environmental Psychology in the Outdoor Environment Design of Kindergarten [J]. Sichuan: Sichuan Architecture, 2010,30 (4): 111-113.
[15] Duan Zhaolin. Experience in Agriculture and Ecological Resource Conservation: Principles and Practices of Urban and Rural Ecological Environment Construction (Edited by Jianjun Tang, et al.). Beijing: China Environmental Science Press, 2004.

[16] Yu Yiwu, Zhang Jianguo, Zhu Quan et al. Planning and Development of Leisure Agriculture Park [M]. Hangzhou Press, 2007.

[17] Guo Huancheng et al. Research on Leisure Agriculture and Rural Tourism Development in Beijing [J] Beijing: Earth Information Science, 2008,10(4):453-46. 